\title{
TCF3 mutually exclusive alternative splicing is controlled by long-range cooperative actions between hnRNPH1 and PTBP1
}

\author{
TAKASHI YAMAZAKI, ${ }^{1}$ LIZHI LIU, and JAMES L. MANLEY \\ Department of Biological Sciences, Columbia University, New York, New York 10027, USA
}

\begin{abstract}
TCF3, also known as E2A, is a well-studied transcription factor that plays an important role in stem cell maintenance and hematopoietic development. The TCF3 gene encodes two related proteins, E12 and E47, which arise from mutually exclusive alternative splicing (MEAS). Since these two proteins have different DNA binding and dimerization domains, this AS event must be strictly regulated to ensure proper isoform ratios. Previously, we found that heterogeneous nuclear ribonucleoprotein (hnRNP) H1/F regulates TCF3 AS by binding to exonic splicing silencers (ESSs) in exon 18b. Here, we identify conserved intronic splicing silencers (ISSs) located between, and far from, the two mutually exclusive exons, and show that they are essential for MEAS. Further, we demonstrate that the hnRNP PTBP1 binds the ISS and is a regulator of TCF3 AS. We also demonstrate that hnRNP H1 and PTBP1 regulate TCF3 AS reciprocally, and that position-dependent interactions between these factors are essential for proper TCF3 MEAS. Our study provides a new model in which MEAS is regulated by cooperative actions of distinct hnRNPs bound to ISSs and ESSs.
\end{abstract}

Keywords: TCF3; alternative splicing; hnRNPH; PTBP1; intronic splicing silencer

\section{INTRODUCTION}

Alternative splicing (AS) is an important mechanism that contributes to the regulation of gene expression and generation of proteomic diversity (Wang et al. 2008; Baralle and Giudice 2017). Whether an alternative exon is included or excluded during splicing is often determined by RNA elements that are recognized by regulatory RNA binding proteins (RBPs) (Chen and Manley 2009). The two groups of classical AS regulators are the hnRNP proteins, which often act as splicing repressors, and serine/arginine-rich (SR) proteins, which usually function as splicing activators. hnRNP proteins bind to exonic splicing silencers (ESSs) or intronic splicing silencers (ISSs) to inhibit splicing, whereas SR proteins function by recognizing exonic splicing enhancers (ESEs). Orchestrating these and/or other splicing regulators/elements results in the major distinct subtypes of AS: exon skipping, intron retention, alternative $3^{\prime} / 5^{\prime}$ splice site usage, and the relatively rare mutually exclusive AS (MEAS).

TCF3, also known as E2A, is a member of the $\mathrm{E}$ box protein (class I) family of HLH transcription factors (Murre et al.

\footnotetext{
${ }^{1}$ Present address: Neuroscience Drug Discovery Unit, Innovative Biology Laboratories, Takeda Pharmaceutical Co. Ltd., Fujisawa, Kanagawa 251-8555, Japan

Corresponding author: jlm2@columbia.edu

Article is online at http://www.rnajournal.org/cgi/doi/10.1261/rna. 072298.119.
}

1989). Many studies have revealed that TCF3 plays important roles in a variety of developmental processes (Bohrer et al. 2015; Cunningham et al. 2017; Miyazaki et al. 2017). The TCF3 gene encodes two major isoforms that arise from MEAS. These isoforms, E12 and E47, are related transcription factors that differ only in their bHLH DNA binding regions and have different dimerization preferences and hence different DNA binding properties (Sun and Baltimore 1991). This can lead to different functional consequences. For example, one study using knockout mice deficient for E12 or E47 revealed that E47 is essential for developmental progression at the prepro-B cell stage, whereas E12 is dispensable for early B cell development, commitment and maintenance (Beck et al. 2009). In cortical neurogenesis, E47 is required for proper neuronal differentiation and layer-specific localization, whereas E12 is dispensable for early corticogenesis (Pfurr et al. 2017). In addition, we recently reported that E12 and E47 differentially regulate expression of $E$ cadherin during human embryonic stem cell (hESC) differentiation (Yamazaki et al. 2018). These reports together suggest that TCF3 MEAS

\footnotetext{
(C) 2019 Yamazaki et al. This article is distributed exclusively by the RNA Society for the first 12 months after the full-issue publication date (see http://rnajournal.cshlp.org/site/misc/terms.xhtml). After 12 months, it is available under a Creative Commons License (Attribution-NonCommercial 4.0 International), as described at http:// creativecommons.org/licenses/by-nc/4.0/.
} 
plays an important role in a variety of developmental processes.

How TCF3 MEAS is regulated is not fully understood. We recently showed that the highly related and developmentally regulated hnRNP $\mathrm{H} 1$ and $\mathrm{F}$ proteins contribute to TCF3 MEAS regulation during hESC differentiation by binding to ESSs in exon 18b of TCF3 (Yamazaki et al. 2018). However, how hnRNP H1/F function and what other RBPs might be involved are unknown. Indeed, as multiple proteins bind the ESSs identified in our previous study (Yamazaki et al. 2018), it is likely that TCF3 MEAS is regulated by additional RBPs. This possibility is also supported by previous studies by our laboratory, which demonstrated that the hnRNPs PTBP1 and hnRNP A1/A2 function together to regulate MEAS of pyruvate kinase $\mathrm{M}$ (PKM) pre-mRNAs (David et al. 2010; Chen et al. 2012).

In this study, we provide additional insights into how TCF3 MEAS is regulated. We identify evolutionarily conserved distal ISSs located between the two mutually exclusive exons and show that they are involved in TCF3 MEAS. RNA affinity assays show that PTBP1 and DDX21, a DEAD-box RNA helicase, bind to these conserved ISSs. We also demonstrate that cooperative actions of PTBP1 bound to these newly identified ISSs and hnRNP H1 bound to ESSs in exon 18b control TCF3 MEAS. Our study thus reveals the details of TCF3 MEAS and suggests a new mechanism of MEAS regulation.

\section{RESULTS}

\section{A distal intronic conserved region is involved in TCF3 MEAS}

As described above, our previous study identified ESSs in exon 18b that are essential for TCF3 MEAS. However, given the complexity of MEAS, we suspected that additional sequence elements and regulatory RBPs are involved in control of TCF3 AS. To gain additional insight into the mechanism of TCF3 MEAS, we first set out to identify additional cis-acting sequence elements in the TCF3 premRNA. As an initial approach, we searched for evolutionary conservation throughout the genomic region spanning exon 17 to exon 19. Intriguingly, a distal intronic conserved region (ICR) was found between exons $18 \mathrm{a}$ and $18 \mathrm{~b}$ [>500 nucleotides ( $\mathrm{nt}$ ) from either exon and $400 \mathrm{nt}$ in length; Fig. 1A]. We hypothesized that this ICR functions as an intronic splicing regulatory element. To test this idea, we used a previously developed TCF3 minigene system that recapitulates TCF3 MEAS changes (Yamazaki et al. 2018). We initially created two mutated TCF3 minigene constructs, ICRdel, in which only the ICR has been deleted, and Intdel, in which the entire intronic region other than the ICR was deleted (Fig. 1B). These constructs and the unmodified parental vector were transfected into HeLa cells and the resultant transcripts were analyzed by semi-quan- titative ${ }^{32} \mathrm{P}$ RT-PCR. As shown in Figure 1C, deletion of the ICR essentially eliminated exon $18 \mathrm{~b}$ inclusion (or increased exon 18a inclusion) and also gave rise to low levels of a double inclusion (DI) product that included both exons. Interestingly, deletion of other parts of the intron did not significantly affect TCF3 splicing (Fig. 1C). These data suggest that the sequences within the ICR are essential for proper TCF3 MEAS.

The conservation analysis described above revealed that this intronic region contains four highly conserved distinct motifs, which we named CE1, CE2, CE3, and CE4 (Figs. 1A, 2A). To determine which motif(s) contain(s) the splicing regulatory element(s), we generated four mutated TCF3 minigenes, each devoid of one of the four motifs (CE18del-1, -2, -3 and -4) (Fig. 2A) and used them in transfection assays as above. We quantified percent exon $18 \mathrm{a}$ inclusion by dividing the exon 18 a signal by the sum of exons $18 \mathrm{a}$ and 18b. (Note that the double-skipped isoform was not included, since for unknown reasons the levels of this species displayed relatively high variation from experiment to experiment, whereas the E12/E47 ratios were much more consistent.) Deletion of CE3 or CE4 sharply reduced exon $18 \mathrm{~b}$ inclusion (percent exon $18 \mathrm{a}$ was increased from $54 \%$ to $93 \%$ or $84 \%$, respectively). Although its effect was relatively mild, CE2 deletion also reduced exon $18 \mathrm{~b}$ inclusion (from 54\% to $70 \%$ ). On the other hand, deletion of CE1 did not affect the splicing pattern (Fig. 2B). This result indicates that CE3 and CE4 contain sequences that are critical for proper TCF3 MEAS.

Since deletion of CE3 and CE4 had strong effects on TCF3 MEAS, we next set out to characterize the CE3 and CE4 elements further. Specifically, we constructed additional minigenes by mutating CE3 or CE4 in 5 nt blocks (Fig. 2C) and analyzed them as above. In this series of mutations, mutants 4 and 5 in CE3 increased exon 18a inclusion, from $52 \%$ to $91 \%$ and $88 \%$, respectively. Increases of exon 18 a inclusion (from $52 \%$ to $83 \%$ and $85 \%$ ) were also observed in mutants 2 and 3 in CE4 (Fig. 2D). These experiments indicate that these two $10 \mathrm{nt}$ motifs, CE3 (TCGCACTTGC) and CE4 (GTTTGGTCTG), constitute intronic regulatory elements that are essential for exon $18 \mathrm{~b}$ inclusion.

\section{DDX21 and PTBP1 bind to the conserved intronic splicing regulatory elements}

Having identified intronic RNA elements that are necessary for exon $18 \mathrm{~b}$ inclusion, we next wished to identify RBPs that bind these sequences. To this end, we performed RNA affinity assays using nuclear extracts (NEs) prepared from HeLa cells and four biotin-labeled 18-nt RNA oligonucleotides containing either the WT CE3 and CE4 regulatory motifs identified above or mutated derivatives (Fig. 3A, left; Fig. 3B, top). Precipitated proteins were 
A

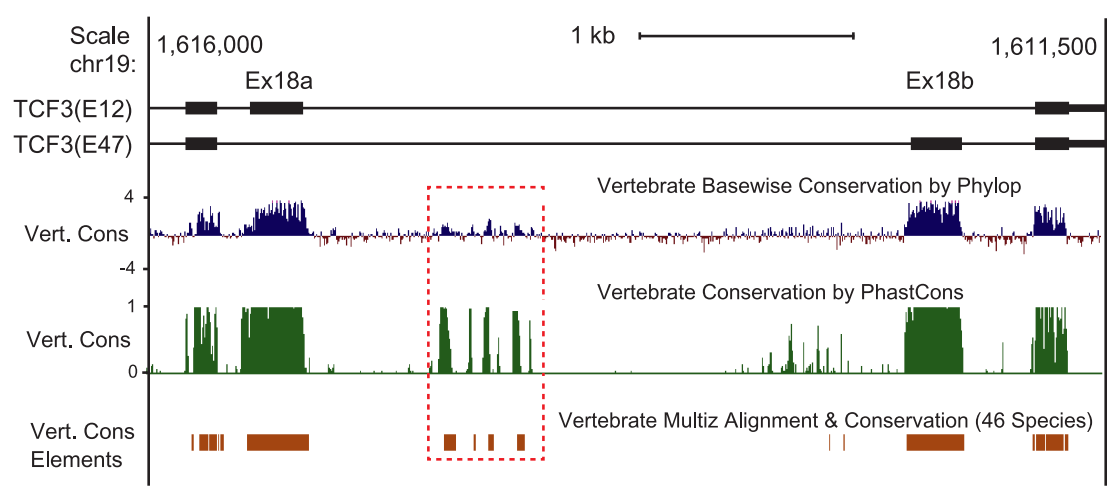

B

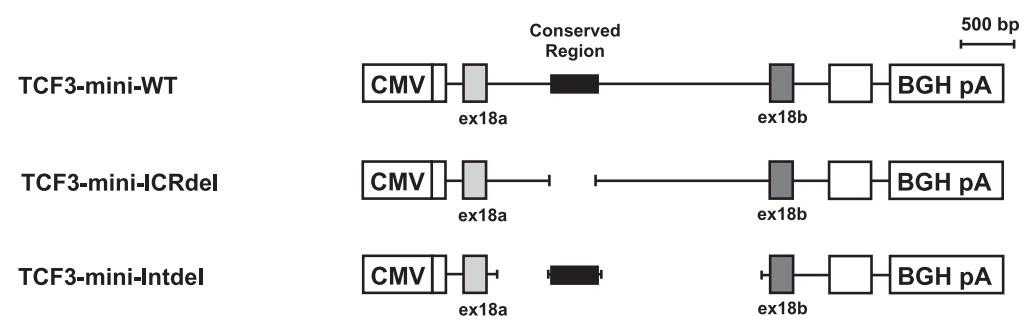

C

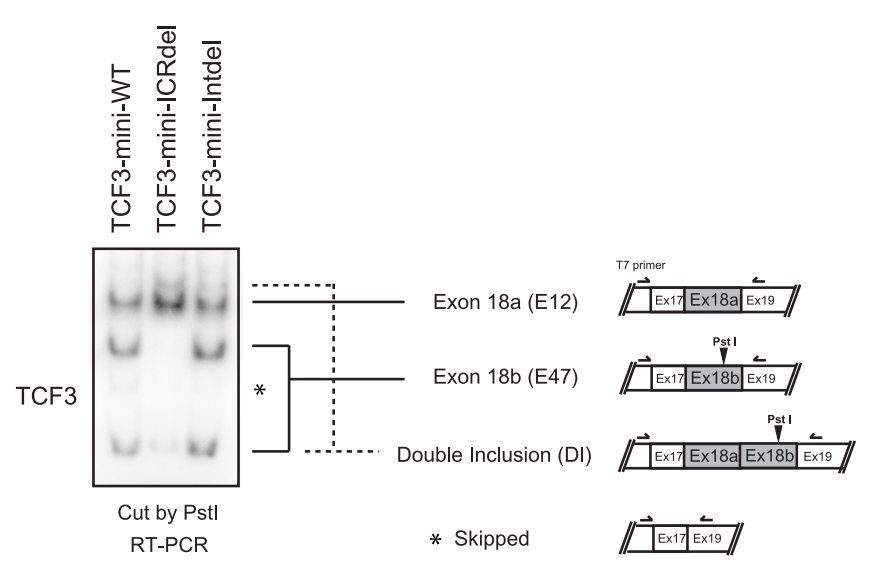

FIGURE 1. The conserved distal intronic region of TCF3 functions as a cis-acting element. (A) Conservation analysis of sequences surrounding TCF3 human exons $18 \mathrm{a}$ and $18 \mathrm{~b}$ across 46 vertebrate species. The TCF3 gene contains a distal ICR between exons 18a and 18b, indicated by the red dashed box. Conservation levels are shown by Phylop and PhastCons. Conserved sequences are shown by Multiz at the bottom. All conservation plots were generated from the UCSC browser using the hg19 genome assembly. (B) Schematic presentations of wild type (WT) and mutated TCF3 minigenes. (C) Splicing of the transfected minigenes (depicted in panel B) in HeLa cells by ${ }^{32}$ P RT-PCR, using T7 primer and Ex19R. PCR products were digested with Pstl and resolved on 5\% PAGE gel. Three independent experiments were performed and the representative result was shown.

resolved by SDS-PAGE and were visualized by silver-staining. This experiment revealed two proteins of molecular weights $\sim 87$ and $55 \mathrm{kDa}$ that bound specifically to WT CE3 and CE4 sequences, respectively, but not to the mutated sequences (Fig. 3A, left; Fig. 3B, left). Mass spectrometry analysis identified the $87 \mathrm{kDa}$ protein as the
DEAD-box protein DDX21 and the $55 \mathrm{kDa}$ protein as the RBP PTBP1. Western blots confirmed the identities of the two proteins and their loss of binding to the mutated sequences (Fig. 3A, right; Fig. 3B, right). These data suggest that DDX21 and PTBP1 function in TCF3 MEAS.

\section{PTBP1 and hnRNPH1 reciprocally regulate TCF3 AS}

Since the above results suggest that DDX21 and PTBP1 are involved in TCF3 MEAS regulation, we next tested whether depletion or overexpression of either protein affects the splicing pattern of TCF3. We first transfected HeLa cells with siRNAs targeting DDX21 or PTBP1 (see Fig. 3C, bottom). siRNA targeting hnRNP $H 1$, which we used in our previous study (Yamazaki et al. 2018), was used as positive control. Consistent with what we reported previously, hnRNP H1 knockdown (KD) increased exon 18b inclusion (exon 18a in total was decreased from $45 \%$ to $28 \%$ ), likely due to loss of repression that resulted from binding the ESSs in exon 18b (Fig. 3C). In contrast to hnRNP H1 KD, PTBP1 KD increased exon 18a inclusion, from $45 \%$ to $53 \%$ (Fig. 3C). Next, we cotransfected HeLa cells with DDX21, PTBP1, or hnRNP $\mathrm{H} 1$ expression vectors together with the WT TCF3 minigene. As we showed in our previous study (Yamazaki et al. 2018), overexpression of hnRNP H1 suppressed exon 18b inclusion (exon 18a inclusion levels increased from $46 \%$ to $65 \%$ ) (Fig. 3D). The resulting overexpression of PTBP1 suppressed exon 18a inclusion, from $46 \%$ to $27 \%$ (Fig. 3D). These results indicate that PTBP1 binding to the ICR we identified represses exon 18a inclusion. Unexpectedly, both depletion and overexpression of DDX21 slightly increased exon 18b inclusion (Fig. 3C,D). While our results together suggest a role for DDX21 in TCF3 MEAS, these and other results suggest that it might be complex. We therefore have not pursued DDX21 function further, but discuss its possible role in TCF3 MEAS below. 
A

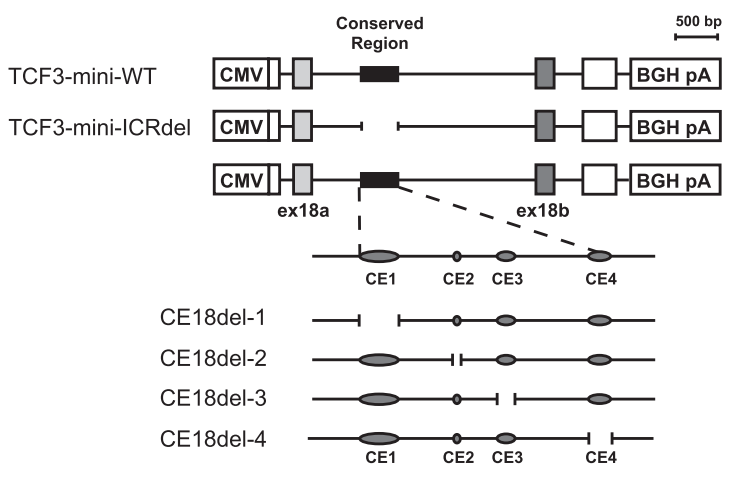

C

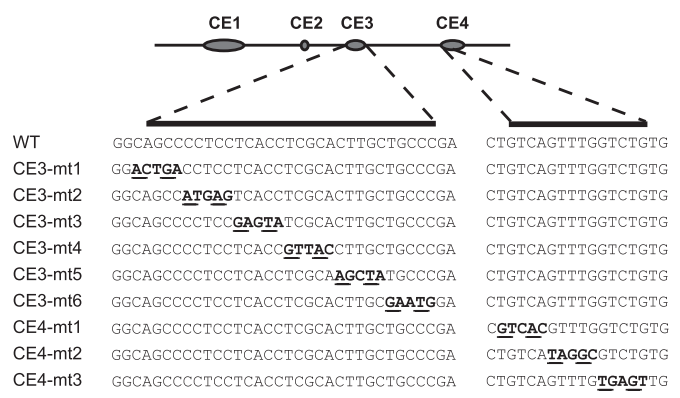

B

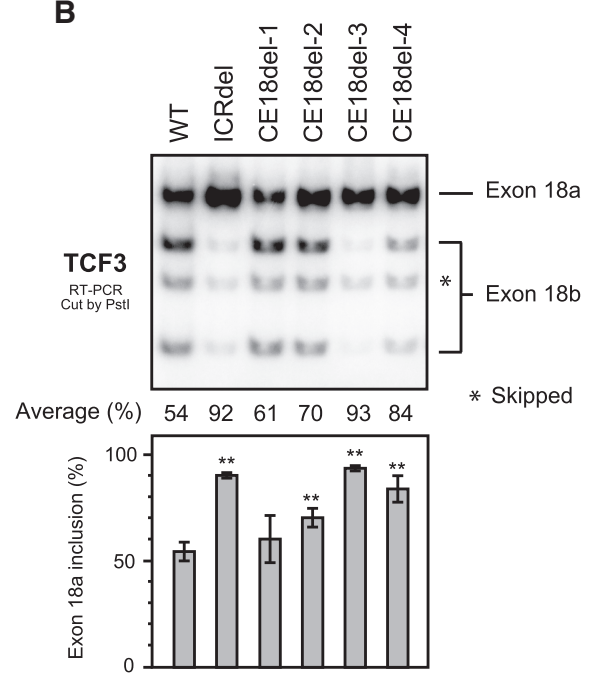

D

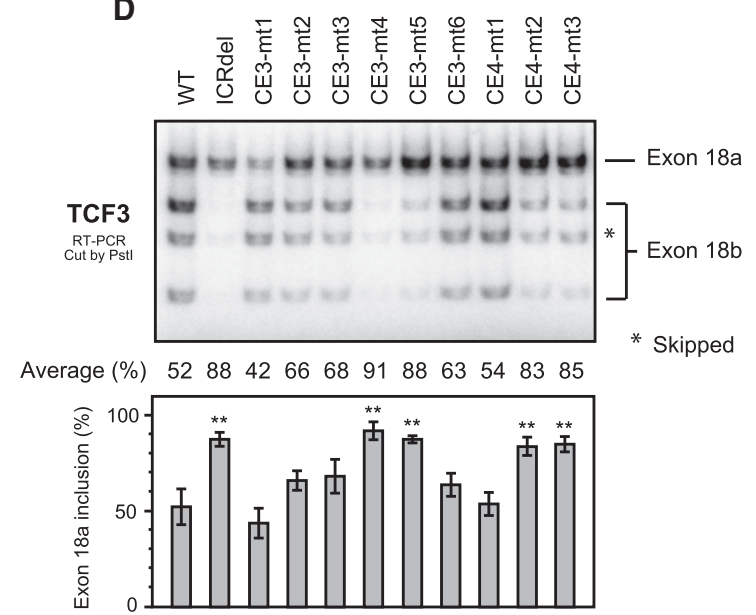

FIGURE 2. Identification of cis-acting elements that mediate TCF3 alternative splicing. (A) Schematic presentations of TCF3 exon-intron structure around the two exon 18s and the minigenes with mutated conserved element (CE). The four distinctive CEs are indicated by gray ovals. (B) Splicing of the transfected TCF3 minigenes (depicted in panel A) in HeLa cells by ${ }^{32}$ P RT-PCR. PCR products were digested by Pstl and resolved on 5\% PAGE gel. (C) Schematic presentations of CE3 and CE4 mutated minigenes. (D) Splicing of the transfected TCF3 minigenes (depicted in panel $C$ ) in HeLa cells by ${ }^{32}$ P RT-PCR. $(B, D)$ The exon 18a inclusion percentages were calculated by dividing exon 18a signal by the sum of exons $18 \mathrm{a}$ and $18 \mathrm{~b}$ signals. The results are indicated in bar graph with means \pm SD from three independent experiments. Averages of $18 \mathrm{a}$ inclusion ratio are indicated on the top. $\left(^{* *}\right) P<0.01$.

\section{The ICR represses either exons $18 \mathrm{a}$ or $18 \mathrm{~b}$}

Our results indicate that deletion of the ICR we identified resulted in decreased $18 \mathrm{~b}$ inclusion and/or increased exon 18a inclusion. To determine whether the ICR functions by repressing exon 18 a or by facilitating exon $18 \mathrm{~b}$ usage, we constructed additional mutated TCF3 minigenes by precisely deleting either exon $18 \mathrm{a}$ or $18 \mathrm{~b}$, with or without ICR deletion (Fig. 4A), and then analyzed splicing as above. Interestingly, simultaneous deletion of exon 18a and the ICR resulted in more exon $18 \mathrm{~b}$ inclusion compared to deletion of exon 18a alone, from $34 \%$ to $77 \%$ (Fig. 4B). However, this phenomenon was also observed following exon $18 \mathrm{~b}$ deletion: Simultaneous deletion of the ICR enhanced exon 18a inclusion, from $41 \%$ to $95 \%$ (Fig. 4B). Thus, rather than targeting a specific exon, the ICR appears to repress inclusion of either exon 18a or exon $18 \mathrm{~b}$. This raises the possibility that interplay between the ICR and the ESSs we identified previously (Yamazaki et al. 2018) may function to control TCF3 MEAS.

\section{Positional relationship of the ESSs and the ICR are essential for TCF3 MEAS}

We next asked how the PTBP1-dependent repressive function of the ICR might cooperate with the hnRNP 
A

CE3-WT: CGCACUUGCUGCCCGAGC CE3-mt: CGCAAGCUAGAAUGGAGC

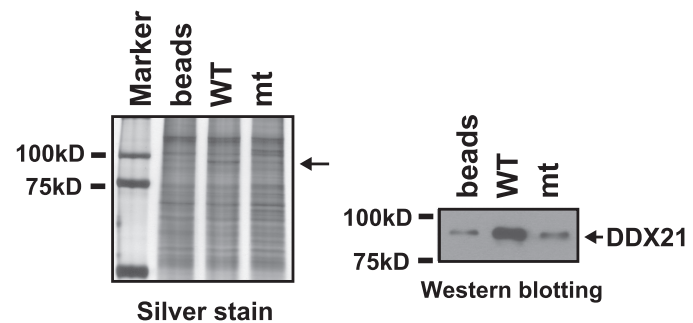

C

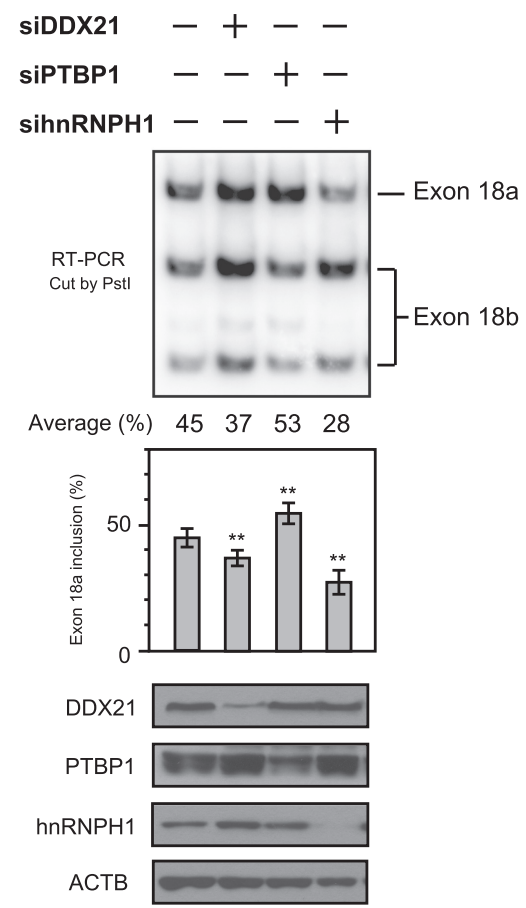

D
B

CE4-WT: CUGUCAGUUUGGUCUGUG CE $4-m t$ : CUGUCAUAGGCUGAGUUG

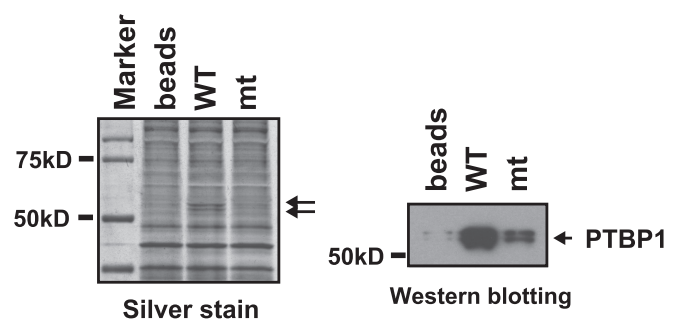

3xFLAG-DDX21v1 -+---
3xFLAG-DDX21v2 --+--
3xFLAG-PTBP1 ---+-
3xFLAG-hnRNPH1 ----+

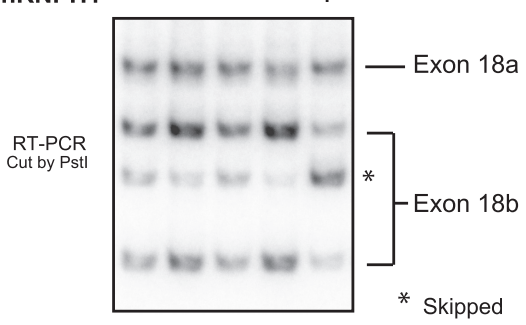

Average (\%) $46 \quad \begin{array}{lllll}39 & 42 & 27 & 65\end{array}$

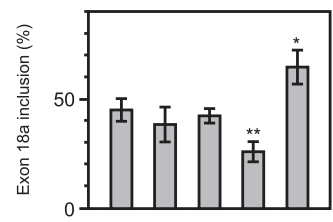

WB: FLAG

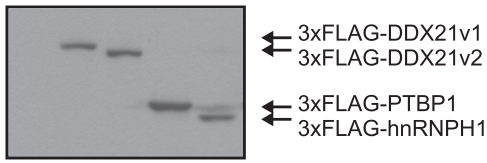

FIGURE 3. PTBP1 and hnRNPH1 reciprocally regulate TCF3 alternative splicing. ( $A, B)$ RNA oligonucleotide sequences of WT and mutated (mt) regulatory motifs from CE3 and CE4 (top). Isolated proteins were resolved on 10\% SDS-PAGE and were stained by silver staining for visualization (bottom left). The indicated bands were analyzed by mass spectrometry. Protein levels were analyzed by western blot using the indicated antibodies (bottom right). (C) Splicing of the endogenous TCF3 in HeLa cells by ${ }^{32}$ P RT-PCR after the indicated siRNA treatments. PCR products were digested by Pstl and resolved by 5\% PAGE. (D) Splicing of the transfected WT TCF3 minigene in HeLa cells by ${ }^{32}$ P RT-PCR using T7 primer and Ex19R after overexpression of the indicated exogenous genes. DDX21v1 and DDX21v2 are isoforms encoded by the DDX21 gene. (C,D) The exon 18a inclusion percentages are calculated by dividing exon 18a signal by the sum of exons 18a and 18b signals. The results are indicated in bar graph with means \pm SD from at least three independent experiments. Averages of $18 \mathrm{a}$ inclusion ratio are indicated on the top. $\left(^{*}\right) P<$ 0.05. (**) $P<0.01$.

H1/F-dependent ESSs in exon 18b to coordinate TCF3 MEAS. Previously, we observed that swapping the positions of the two ME exons in a TCF3 minigene resulted in the exclusive inclusion of exon 18a, whereas the inclusion ratio of exon $18 \mathrm{a}$ to $18 \mathrm{~b}$ was approximately $1: 1$ in the WT construct (Yamazaki et al. 2018).

In light of our current results, we considered the possibility that there might be a position-dependent interaction between the ICR and the ESSs. To test this idea, we generated additional mutated TCF3 minigenes with duplicated exon $18 \mathrm{a}$ or duplicated exon $18 \mathrm{~b}$, by replacing exon $18 \mathrm{~b}$ with exon 18a, or vice versa (Fig. 5A). To differentiate the two repeated exons, a Pstl site was either introduced into the second exon or removed from the first exon by a single nt substitution (CTCCAC to CTGCAG to introduce, or vice versa, to remove). These minigenes thus contain or lack the hnRNP H1/F ESSs in each duplicated exon 18. Because the two exons are highly similar (74\% identical) and the two ESSs in exon 18b are the only functional differences between the two, these constructs allow us to 
A

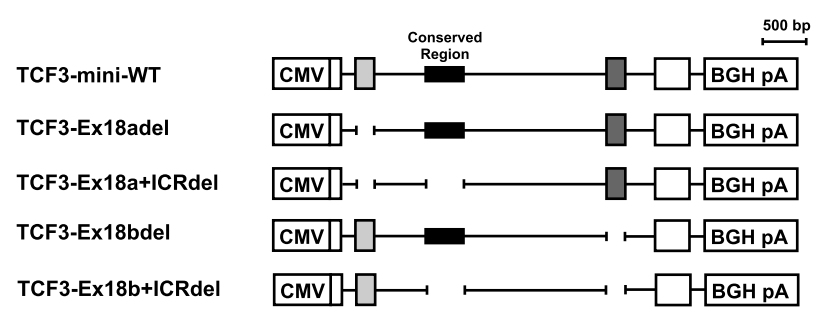

B
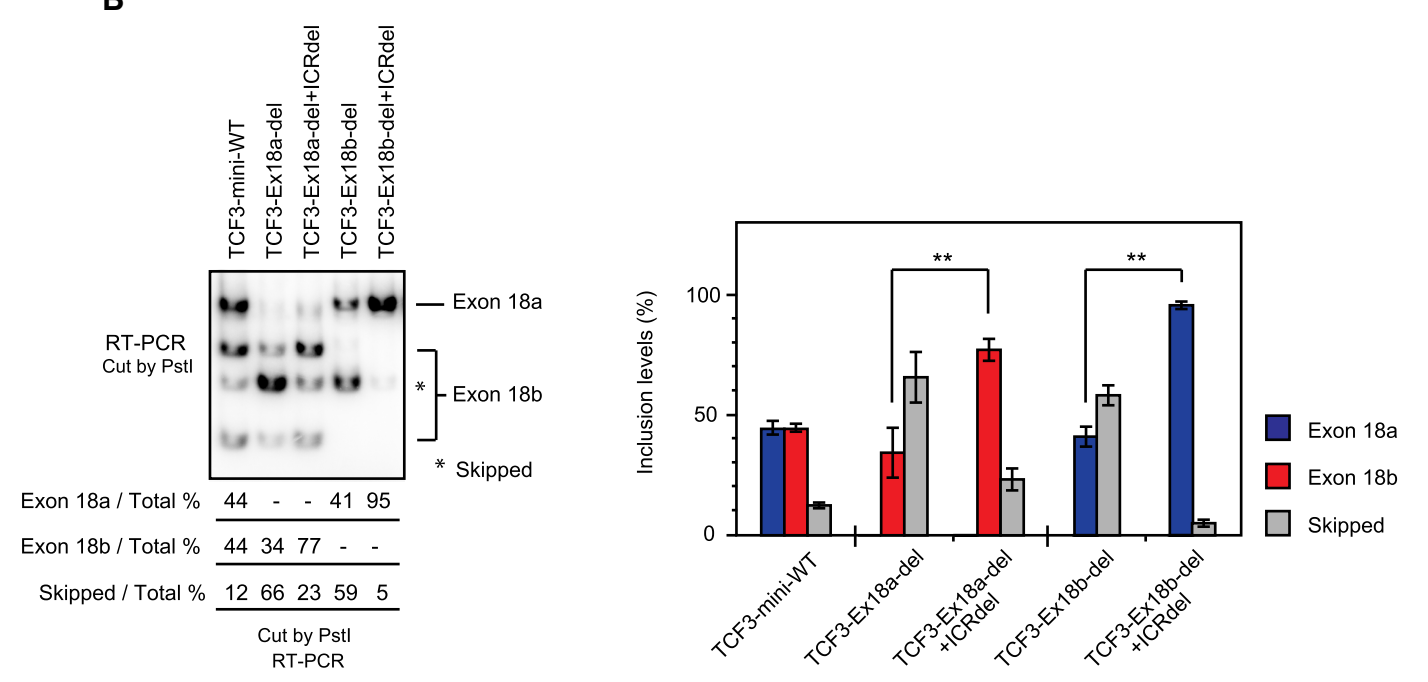

FIGURE 4. The conserved intronic elements act as splicing silencers against both exons 18a and 18b. (A) Schematic presentations of mutated TCF3 minigenes with one of the two exon 18s deleted, with or without ICR deletion. (B) Splicing of the transfected TCF3 minigenes (depicted in panel A) in HeLa by ${ }^{32}$ P RT-PCR, using T7 primer and Ex19R. PCR products were digested with Pstl. The percentages of all spliced are indicated in bar graph with means \pm SD from three independent experiments. (**) $P<0.01$. Averages are indicated at the bottom of the RT-PCR image.

determine the effects of the ESSs in a position-dependent manner. The mutated minigenes were transfected into HeLa cells and splicing patterns were determined by RTPCR. The results showed that the second (distal) exon 18a, located in the original exon $18 \mathrm{~b}$ position, was exclusively included with the duplicated exon 18a minigene (Fig. 5B, lanes 1 in both panels). This finding is consistent with our previous results that mutation or deletion of the ESSs in exon $18 \mathrm{~b}$ strongly increased exon 18b inclusion (Yamazaki et al. 2018). On the other hand, in addition to exclusive inclusion of the second exon 18b, we observed a substantial amount of the double-skipped form with the duplicated exon 18b minigene (Fig. 5B, lanes 3 in both panels).

We next wished to examine the effect of the ICR in the context of the duplicated exons containing or lacking the ESSs. To this end, we deleted the ICR from the double exon 18a/b minigenes and analyzed splicing as above (Fig. 5A). Strikingly, deletion of the ICR in the duplicated exon 18a minigene gave rise to a substantial amount of the DI form, in contrast to the inclusion of only the distal exon 18a observed in the presence of the ICR (Fig. 5B, lanes 2 in both panels). This result supports the idea that the ICR functions to prevent exon 18a inclusion, although the
ICR's repressive function is not limited to this exon (see Fig. 4), and is also consistent with our finding that reduced levels of PTBP1, which binds to the ICR, increased exon 18a inclusion (Fig. 3). Furthermore, deletion of the ICR in the duplicated exon 18a minigene prevented single inclusion of the first (natural) exon 18a (Fig. 5B). This result indicates that the ESSs in exon $18 \mathrm{~b}$ are essential for the sole inclusion of exon 18a. In addition, the finding that deleting the ICR created the DI form, not only in the duplicated exon 18a construct but also in the WT minigene, indicates that the repressive function of the ICR is important to prevent $\mathrm{DI}$ even when the ESSs are present in exon 18b. In contrast to the duplicated exon 18a minigenes, removing the ICR from the double exon $18 \mathrm{~b}$ minigene resulted in the same splicing pattern as that of the same minigene with the ICR (Fig. 5, lanes 3 and 4 in both panels). Since these two duplicated exon $18 \mathrm{~b}$ constructs both have exon $18 \mathrm{~b}$ in the original exon 18a position, the presence of the additional ESSs likely completely repressed inclusion of the first exon 18b. Taken together, our data indicate that positiondependent cooperative actions of the ICR and the ESSs are essential for TCF3 MEAS. We discuss below more details of the underlying mechanism. 
A
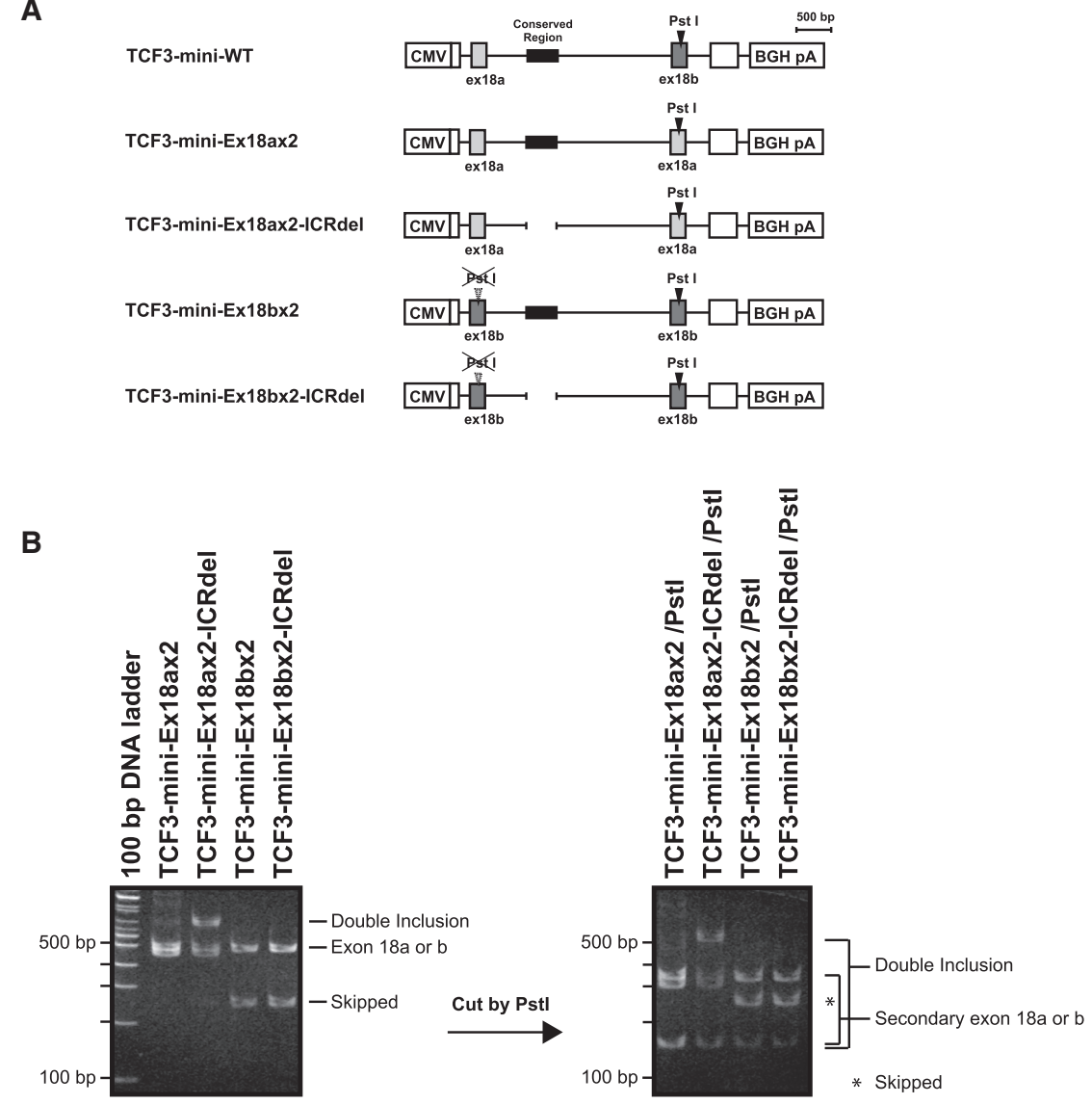

FIGURE 5. The conserved intronic elements and the exonic silencers prevent DI of the two exon 18s. (A) Schematic presentations of mutated TCF3 minigenes containing double exon $18 \mathrm{a}$ or double exon 18b, with or without ICR deletion. (B) Splicing of the transfected TCF3 minigenes (depicted in panel A) in HeLa by RT-PCR, using T7 primer and Ex19R. PCR products were resolved by 5\% PAGE before digestion (left) and after digestion with Pstl (right). essential for proper MEAS regulation. Below we discuss these new insights into the molecular mechanism of TCF3 MEAS in more detail, as well as the implications of our results with respect to alternative splicing control more generally.

The main finding of our work is that bindings of the ESSs by hnRNP $\mathrm{H} 1$ and the ICR by PTBP1 cooperate in TCF3 MEAS, in a manner regulated by the intracellular levels of the two proteins. Based on our data, we propose the following two-step model for control of TCF3 MEAS (Fig. 6). The first step is binding of PTBP1 to the ICR (Fig. 6A). Even though the ICR's repressive function need not be specific to exon 18a, our observations that removing it leads to single inclusion of exon 18a or to the production of the DI form suggests that the natural target of the ICR is exon 18a. This may be simply because exon $18 a$ is transcribed first and exon or intron definition would be prevented by ICR-bound PTBP1 until exon $18 \mathrm{~b}$ is transcribed. Supporting this view, multiple studies have shown that PTBP1 indeed induces skipping of exons by inhibiting exon and intron definition (Wagner and Garcia-Blanco 2001; Izquierdo et al. 2005; Sharma et al. 2005, 2008, 2011; Wongpalee

\section{DISCUSSION}

TCF3 MEAS was first described $30 \mathrm{yr}$ ago (Murre et al. 1989). Since then, accumulating evidence has suggested that this process has a great impact on multiple biological processes. However, how TCF3 MEAS is controlled has not been fully elucidated. Recently, we found that hnRNP H1/F regulates TCF3 isoform expression by binding to ESSs in exon $18 b$ in hESCs (Yamazaki et al. 2018). In this study, we provide additional insights into how this process is regulated. We showed that evolutionarily conserved ISSs (which we refer to as the ICR) located between the two mutually exclusive exons are essential for proper TCF3 MEAS. RNA affinity purification showed that PTBP1 and DDX21 bind the ICR, and functional assays showed that PTBP1 and hnRNP $\mathrm{H} 1$ determine which exon is included in a reciprocal manner. That is, when PTBP1 levels are high exon $18 \mathrm{~b}$ is preferentially included, whereas when hnRNP H1 levels are high exon 18a is included. In addition, our data indicate that position-dependent cooperative interactions between PTBP1/ICR and hnRNP H1/ESSs are et al. 2016). The second step is selection of exon 18a or $b$, which depends on hnRNP $\mathrm{H} 1$ availability. When hnRNP H1 levels are low, the inclusion of exon $18 \mathrm{~b}$ is favored because exon 18a is repressed by PTBP1 bound to the ICR (Fig. 6B). Conversely, when highly expressed, hnRNP $\mathrm{H} 1$ binds to the ESSs in exon $18 \mathrm{~b}$ and represses $18 \mathrm{~b}$ inclusion. We suspect that hnRNP H1 might "transfer" the repressive effect of the ICR from exon 18a to exon $18 \mathrm{~b}$ by physically interacting with PTBP1 under the above conditions (Fig. 6C). This idea arose from our data that ICR deletion increases not only single inclusion of exon $18 \mathrm{a}$ but also DI, suggesting that TCF3 AS would not be mutually exclusive if ICR function is solely suppression of exon 18a. The fact that the repressive function of the ICR does not target specific exon 18a or b sequences also supports this model. And importantly, multiple studies have pointed to the existence of physical, cooperative interactions between hnRNP $\mathrm{H} 1$ and PTBP1 (Min et al. 1995; Chou et al. 1999; Markovtsov et al. 2000; Rooke et al. 2003). Together, this mode of action successfully explains the mutually exclusive nature of TCF3 AS. 
A

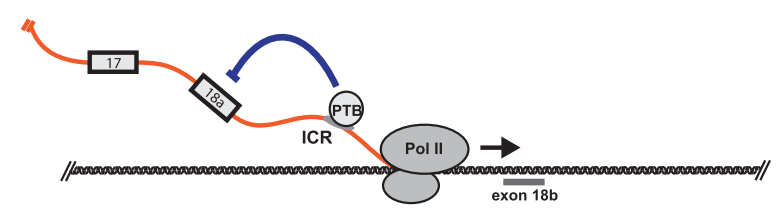

B
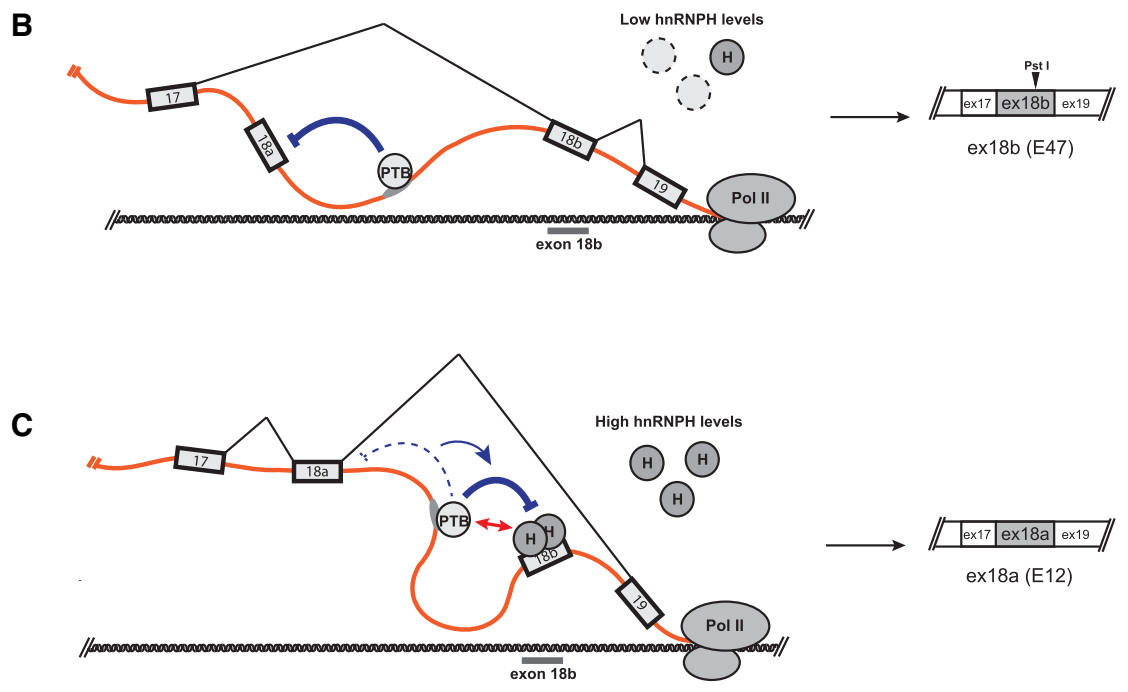

FIGURE 6. A model for TCF3 mutually exclusive alternative splicing. (A) Exon 18a is usually repressed by PTBP1 bound to the ICR. (B) Exon 18b is included under low hnRNP H1 level, because exon 18a is excluded by PTBP1. (C) Exon 18a is included under high hnRNP H1 level, because exon $18 \mathrm{~b}$ is repressed by hnRNP H1 and the repressive function of the ICR is transferred to exon 18b (blue arrow) by physical interaction between PTBP1 and hnRNP H1 (red double headed arrow).

Distinct mechanisms of MEAS regulation have been documented in previous studies. Early work with $\alpha$-tropomyosin pre-mRNA revealed that an unusual far upstream branch site utilized by the downstream ME exon (exon 3) is incompatible with splicing of the upstream exon (Smith and Nadal-Ginard 1989), leading to selective use of exon 3. Exon 2 usage is induced by repression of exon 3, which interestingly is mediated by PTB binding to repressive elements around that exon (Gooding et al. 1998). Our laboratory previously showed that PKM MEAS is controlled by PTBP1 and hnRNP A1/A2 in a concentration-dependent manner (David et al. 2010; Chen et al. 2012). However, the TCF3 MEAS mechanism revealed by the current study is different from the PKM MEAS mechanism, even though PTBP1 functions in both. For example, inclusion or exclusion of one of the two ME exons in PKM AS is independent of exon position and is dominantly determined by ESSs in exon 9 (the upstream exon), whereas TCF3 MEAS is dependent on the position of the two exons for proper cooperative actions between the ESSs and ICR. Additionally, PKM MEAS requires many cis-elements in the ME exons and flanking introns, while TCF3 MEAS appears to be mostly regulated by the ESSs in exon $18 \mathrm{~b}$ and the ICR. These differ- ences in regulatory mechanisms between different MEAS events provide important insights into how interactions between multiple splicing factors/elements create diverse modes of AS regulation. Interestingly, PTBP1, hnRNP H1, and hnRNP A have been implicated in the regulation of multiple MEAS events (Llorian et al. 2010; Huelga et al. 2012). It will be interesting to learn how widely each of the above mechanisms, or related ones, is/are used for MEAS regulation.

An additional question is how DDX21 is involved in TCF3 MEAS. DDX21 has been implicated in transcription and ribosomal RNA processing (Calo et al. 2015), but its functions in splicing regulation have not been reported. As with other DDX proteins that are known to function in premRNA splicing (e.g., DDX5, DDX17, DDX23, DDX39; Mathew et al. 2008; Dardenne et al. 2014; Nakata et al. 2017; Lee et al. 2018), DDX21 also has RNA helicases activity (Gustafson and Wessel 2010). In addition, DDX21 indeed copurified with hnRNP $\mathrm{H} 1$ and PTBP1 in messenger ribonucleoprotein (mRNP) complexes isolated using an hnRNP A antibody (Close et al. 2012), and was isolated with RNA oligonucleotides corresponding to a characterized exonic splicing regulatory sequence (Hall et al. 2013). These findings support the view that DDX21 functions in some way in splicing regulation. However, our gain and loss of functions experiments did not clarify its function. Either RNAi-mediated depletion or overexpression of DDX21 resulted in a very modest increase in exon 18b inclusion. Interestingly, and consistent with our findings, data from the ENCODE project (https://www.encodeproject.org) showed that DDX21 KD did not have strong effects on the TCF3 MEAS, in HepG2 and K562 cells, although DDX21 binding was detected on the ICR (precisely on CE3) by eCLIP in $\mathrm{K} 562$ cells. One possible explanation for these results is that when DDX21 is depleted with RNAi, another DDX family member may function redundantly, and this may in fact mimic the DDX21 overexpression effect we observed.

Another interesting question is how might DDX21 function in TCF3 MEAS. Interestingly, a recent study revealed that DDX21 binds and unwinds RNA G-quadruplex structures (G-Os) (McRae et al. 2017). G-Os are known to be an important secondary structure formed from some G-rich sequences and are involved in multiple gene expression steps including alternative splicing (Verma and 
Das 2018; Weldon et al. 2018). Furthermore, hnRNP H1 is known to bind RNAs that form G-Qs although it displays somewhat higher affinity when the ability of the RNA to form G-Qs is reduced (Conlon et al. 2016). Notably, both ESSs in exon18b, which are recognized by hnRNP H1, are predicted to form G-Qs, as simulated by QGRS mapper (Kikin et al. 2006). Thus, an attractive idea is that DDX21 is involved in TCF3 MEAS by modulating RNA structure to facilitate hnRNP H1 binding.

In addition to the above possibility, the RNA helicase activity of DDX21 is interesting with respect to possible regulatory mechanisms used by distal intronic cis-elements. Although previous studies have described examples of AS regulation with distal intronic regulatory sequences, the mechanisms by which a splicing factor might act on a distant exon have been largely obscure (Guo and Kawamoto 2000; Coté et al. 2001; Baraniak et al. 2003; Dirksen et al. 2003; Lenasi et al. 2006). Interestingly, however, a relatively recent genome-wide study on Rbfox has shown that more than half of Rbfox binding sites that are conserved through evolution in mammalian brain are located distally (>500 nt) from exons, and these intronic elements affect distal exon inclusion by modulating the distance between these elements, affecting exons via long-range RNA-RNA secondary bridge structures (Lovci et al. 2013). The ICR we identified in TCF3 AS has similar characteristics with such distal cis-elements, that is, it is located $>500$ nt from either exon and evolutionally conserved, at least in mammals. Interestingly, our results showed that deletion of all intronic sequences between exons 18a and b except for the ICR slightly increased production of the $\mathrm{DI}$ isoform, consistent with a possible role for long-range interactions in ensuring accurate exon inclusion. While our data has shown that the primary regulatory mechanism for TCF3 MEAS involves interactions between the ESSs/hnRNP H1 and ISSs/PTBP1, it may also be that RNA-RNA secondary bridge structures function in TCF3 MEAS. This suggests another possible function for DDX21 in modulating RNA-RNA secondary bridge structures. In any case, it will be interesting to determine the exact function of DDX21 in future studies.

As indicated above, we found that the ratio of PTBP1 and hnRNP $\mathrm{H} 1$ expression levels is important for control of TCF3 MEAS. An intriguing question then is how do levels of these RBPs change during TCF3 AS-related developmental processes. We previously observed the switching of TCF3 MEAS from exon 18a to 18b during hESC differentiation reflected a corresponding decrease in hnRNP H1/F levels (Yamazaki et al. 2018). We speculated that the ratio of PTBP1/hnRNP H/F would increase during hESC differentiation, in keeping with this switch in splicing. However, our data (not shown) suggests a small decrease in PTBP1 and only very modest changes in this ratio, likely insufficient by itself to drive the TCF3 MEAS switch. One possible explanation for this is that tissue-specific paralogs of PTBP1 (such as nPTB and ROD1), which have some redundant functions with PTBP1 (Spellman et al. 2007), increase during differentiation. Indeed, studies have shown that nPTB levels do increase when PTBP1 levels decrease during neural differentiation (Boutz et al. 2007; Linares et al. 2015). This might serve to increase the PTB/hnRNP $\mathrm{H} / \mathrm{F}$ ratio, and thereby drive the exon $18 \mathrm{a}$ to $18 \mathrm{~b}$ switch. Further investigation will be required to understand how PTBP1 and hnRNP H1/F, perhaps with the help of paralogs such as nPTB and hnRNP H2, function together to control TCF3 MEAS.

In conclusion, we have provided detailed insights into the mechanism of TCF3 MEAS. We found that this process is regulated by interactions between PTBP1 bound to an ICR containing ISS elements and hnRNP H1 bound to ESSs in one of the two ME exons, and that a DEAD-box helicase that binds specifically to an ISS in the same ICR may facilitate MEAS by an unknown mechanism.

\section{MATERIALS AND METHODS}

\section{Plasmid constructs}

hnRNP H1 and hnRNP F cDNAs were amplified from HeLa cDNA and cloned into pcDNA3 (Invitrogen) with $3 \times$ Flag-tag using either $\mathrm{BamHI}$ and $\mathrm{Xbal}$ or $\mathrm{BamH} 1$ and Xhol for mammalian expression. Construction of TCF3 minigene reporters is described in Yamazaki et al. (2018). All mutations of TCF3 minigene were introduced by PCR-based site-directed mutagenesis as previously described (Yamazaki et al. 2018). Primer sequences are available upon requests.

\section{RT-PCR and ${ }^{32}$ P RT-PCR}

Total RNA was extracted from tissue culture samples using the TRIzol (Invitrogen) according to the manufacturer's instructions. Total RNA $(1 \mu \mathrm{g})$ was reverse-transcribed using Maxima RT (Thermo Scientific) primed with random hexamers (Invitrogen). RT-PCR was performed using Taq DNA polymerase (Invitrogen). For ${ }^{32} \mathrm{P}$ RT-PCR, $3 \mu \mathrm{Ci}\left[{ }^{32} \mathrm{P}\right] \mathrm{dCTP}$ was added per $50 \mu \mathrm{L}$ PCR reaction. Primers used in the $P C R$ reactions were described in Yamazaki et al. (2018).

\section{Minigene splicing assays}

One hundred nanograms of WT or mutated minigene plasmids were transfected into HeLa cells. To examine the effects of exogenous expression of PTBP1, hnRNP H1, 500 ng of empty vector and/or expression vectors were transfected with $50 \mathrm{ng}$ of TCF3 minigene. Forty-eight hours after transfection, cells were collected and the E12/E47 ratio was analyzed using ${ }^{32}$ P RT-PCR followed by Pstl digestion. Digested PCR products were separated on a $5 \%$ PAGE gel. ImageQuant TL (GE healthcare) was used for quantification. 


\section{Western blotting}

Cells were lysed with SDS sample buffer (50 mM Tris pH 7.4, 100 $\mathrm{mM} \mathrm{NaCl}, 1 \%$ Triton $\mathrm{X}-100,0.1 \%$ SDS, $1 \%$ sodium deoxycholate, and $1 \times$ proteinase inhibitor cocktail [Biotools]) and resolved by SDS-PAGE. Proteins were then transferred to nitrocellulose membranes and incubated with primary antibodies detecting PTBP1 (BB7) (Sigma, 1:8000), DDX21 (Bethyl Laboratories, 1:1000), hnRNPH1 (Bethyl Laboratories, 1:1500), or ACTB (Sigma, $1: 30,000)$ overnight at $4^{\circ} \mathrm{C}$. Secondary HRP-conjugated antimouse and anti-rabbit lgGs (Sigma) were used at 1:10,000 for $2 \mathrm{~h}$ at room temperature. Following washes with PBST $(1 \times$ PBS, $0.1 \%$ Tween-20), ECL-Prime (GE Healthcare) was used to visualize the chemiluminescence signal, and Image J software was used for quantification.

\section{RNA affinity purification}

The $5^{\prime}$ biotinylated 18 RNA oligonucleotides were purchased from IDT. NEs from HeLa cells were prepared as previously described (Kleiman and Manley 2001). Three nanomoles biotinylated RNA were incubated with $100 \mu \mathrm{L}$ of streptavidin-agarose beads (Sigma) overnight at $4^{\circ} \mathrm{C}$ with rotation. Incubated beads were then washed with binding buffer $(10 \mathrm{mM}$ Tris $\mathrm{pH} 7.4,0.5$ $\mathrm{mM}$ EDTA, $500 \mathrm{mM} \mathrm{NaCl}$ ) twice and buffer D (20 mM Hepes pH7.9, 20\% glycerol, $300 \mathrm{mM} \mathrm{KCl}, 0.2 \mathrm{mM}$ EDTA, $0.5 \mathrm{mM}$ DTT) twice. RNA coated beads were then mixed and rotated with NEs from HeLa cells for $4 \mathrm{~h}$ at $4^{\circ} \mathrm{C}$. After washing beads with buffer $D$ twice and with buffer $D$ without glycerol twice, precipitated proteins were eluted by SDS sample buffer and analyzed by WB.

\section{Silver staining}

Isolated proteins were resolved with SDS-PAGE. Gels were fixed by soaking with fixation buffer ( $40 \%$ ethanol, $10 \%$ acetic acid) for $30 \mathrm{~min}$. Fixed gels were soaked with sensitizing buffer $(0.02 \%$ sodium thiosulfate, $0.8 \mathrm{M}$ sodium acetate, $0.1 \%$ glutaraldehyde, $30 \%$ ethanol) for $30 \mathrm{~min}$. After washing with water for $5 \mathrm{~min}$ three times, gels were soaked with reaction buffer $(0.2 \%$ silver nitrate, $0.04 \%$ formaldehyde) for $30 \mathrm{~min}$. Gels were next quickly rinsed with water, then soaked with developing buffer $(2 \%$ sodium carbonate, $0.04 \%$ formaldehyde) for $10 \mathrm{~min}$. The developing step was stopped by soaking with $0.05 \mathrm{M}$ EDTA solution (pH 8.0).

\section{ACKNOWLEDGMENTS}

We thank Amr Al-Zain for help with RT-PCR and members of the Manley laboratory for discussions. We also thank Lewis Brown for help with mass spectrometry. This work was supported by US National Institutes of Health (NIH) R35 GM118136 (J.L.M.). L.L. is supported by the National Science Foundation Graduate Research Fellowship Program, grant no. DGE 1644869.

Received June 12, 2019; accepted July 31, 2019.

\section{REFERENCES}

Baralle FE, Giudice J. 2017. Alternative splicing as a regulator of development and tissue identity. Nat Rev Mol Cell Biol 18: 437451. doi:10.1038/nrm.2017.27

Baraniak AP, Lasda EL, Wagner EJ, Garcia-Blanco MA. 2003. A stem structure in fibroblast growth factor receptor 2 transcripts mediates cell-type-specific splicing by approximating intronic control elements. Mol Cell Biol 23: 9327-9337. doi:10.1128/MCB.23.24 .9327-9337.2003

Beck K, Peak MM, Ota T, Nemazee D, Murre C. 2009. Distinct roles for $\mathrm{E} 12$ and $\mathrm{E} 47$ in $\mathrm{B}$ cell specification and the sequential rearrangement of immunoglobulin light chain loci. J Exp Med 206: 22712284. doi:10.1084/jem.20090756

Bohrer C, Pfurr S, Mammadzada K, Schildge S, Plappert L, Hils M, Pous L, Rauch KS, Dumit VI, Pfeifer D, et al. 2015. The balance of Id 3 and E47 determines neural stem/precursor cell differentiation into astrocytes. EMBO J 34: 2804-2819. doi:10.15252/embj .201591118

Boutz PL, Stoilov P, Li Q, Lin C-H, Chawla G, Ostrow K, Shiue L, Ares M, Black DL. 2007. A post-transcriptional regulatory switch in polypyrimidine tract-binding proteins reprograms alternative splicing in developing neurons. Genes Dev 21: 1636-1652. doi:10.1101/gad.1558107

Calo E, Flynn RA, Martin L, Spitale RC, Chang HY, Wysocka J. 2015. RNA helicase DDX21 coordinates transcription and ribosomal RNA processing. Nature 518: 249-253. doi:10.1038/ nature13923

Chen M, Manley JL. 2009. Mechanisms of alternative splicing regulation: insights from molecular and genomics approaches. Nat Rev Mol Cell Biol 10: 741-754. doi:10.1038/nrm2777

Chen M, David CJ, Manley JL. 2012. Concentration-dependent control of pyruvate kinase M mutually exclusive splicing by hnRNP proteins. Nat Struct Mol Biol 19: 346-354. doi:10.1038/nsmb .2219

Chou MY, Rooke N, Turck CW, Black DL. 1999. hnRNP H is a component of a splicing enhancer complex that activates a c-src alternative exon in neuronal cells. Mol Cell Biol 19: 69-77. doi:10.1128/ MCB.19.1.69

Close P, East P, Dirac-Svejstrup AB, Hartmann H, Heron M, Maslen S, Chariot A, Söding J, Skehel M, Svejstrup JQ. 2012. DBIRD complex integrates alternative mRNA splicing with RNA polymerase II transcript elongation. Nature 484: 386-389. doi:10.1038/ nature 10925

Conlon EG, Lu L, Sharma A, Yamazaki T, Tang T, Shneider NA, Manley JL. 2016. The C9ORF72 GGGGCC expansion forms RNA G-quadruplex inclusions and sequesters hnRNP $H$ to disrupt splicing in ALS brains. Elife 5: e17820. doi:10.7554/eLife .17820

Coté J, Dupuis S, Jiang Z, Wu JY. 2001. Caspase-2 pre-mRNA alternative splicing: identification of an intronic element containing a decoy 3' acceptor site. Proc Natl Acad Sci 98: 938-943. doi:10 $.1073 /$ pnas. 98.3.938

Cunningham TJ, Yu MS, McKeithan WL, Spiering S, Carrette F, Huang C-T, Bushway PJ, Tierney M, Albini S, Giacca M, et al. 2017. Id genes are essential for early heart formation. Genes Dev 31: 1325-1338. doi:10.1101/gad.300400.117

Dardenne E, Polay Espinoza M, Fattet L, Germann S, Lambert MP, Neil H, Zonta E, Mortada H, Gratadou L, Deygas M, et al. 2014. RNA helicases DDX5 and DDX17 dynamically orchestrate transcription, miRNA, and splicing programs in cell differentiation. Cell Rep 7: 1900-1913. doi:10.1016/j.celrep.2014 .05 .010

David CJ, Chen M, Assanah M, Canoll P, Manley JL. 2010. HnRNP proteins controlled by c-Myc deregulate pyruvate kinase mRNA 
splicing in cancer. Nature 463: 364-368. doi:10.1038/ nature08697

Dirksen WP, Mohamed SA, Fisher SA. 2003. Splicing of a myosin phosphatase targeting subunit 1 alternative exon is regulated by intronic cis-elements and a novel bipartite exonic enhancer/ silencer element. J Biol Chem 278: 9722-9732. doi:10.1074/jbc .M207969200

Gooding C, Roberts GC, Smith CW. 1998. Role of an inhibitory pyrimidine element and polypyrimidine tract binding protein in repression of a regulated $\alpha$-tropomyosin exon. RNA 4: 85100.

Guo N, Kawamoto S. 2000. An intronic downstream enhancer promotes $3^{\prime}$ splice site usage of a neural cell-specific exon. J Biol Chem 275: 33641-33649. doi:10.1074/jbc.M005597200

Gustafson EA, Wessel GM. 2010. DEAD-box helicases: posttranslational regulation and function. Biochem Biophys Res Commun 395: 1-6. doi:10.1016/j.bbrc.2010.02.172

Hall MP, Nagel RJ, Fagg WS, Shiue L, Cline MS, Perriman RJ, Donohue JP, Ares M. 2013. Quaking and PTB control overlapping splicing regulatory networks during muscle cell differentiation. RNA 19: 627-638. doi:10.1261/rna.038422.113

Huelga SC, Vu AQ, Arnold JD, Liang TY, Liu PP, Yan BY, Donohue JP, Shiue L, Hoon S, Brenner S, et al. 2012. Integrative genome-wide analysis reveals cooperative regulation of alternative splicing by hnRNP proteins. Cell Rep 1: 167-178. doi:10.1016/j.celrep.2012 .02 .001

Izquierdo JM, Majós N, Bonnal S, Martínez C, Castelo R, Guigó R, Bilbao D, Valcárcel J. 2005. Regulation of Fas alternative splicing by antagonistic effects of TIA-1 and PTB on exon definition. Mol Cell 19: 475-484. doi:10.1016/j.molcel.2005.06.015

Kikin O, D'Antonio L, Bagga PS. 2006. QGRS Mapper: a webbased server for predicting G-quadruplexes in nucleotide sequences. Nucleic Acids Res 34: W676-W682. doi:10.1093/nar/ gkl253

Kleiman FE, Manley JL. 2001. The BARD1-CstF-50 interaction links mRNA $3^{\prime}$ end formation to DNA damage and tumor suppression. Cell 104: 743-753. doi:10.1016/S0092-8674(01)00270-7

Lee YJ, Wang Q, Rio DC. 2018. Coordinate regulation of alternative pre-mRNA splicing events by the human RNA chaperone proteins hnRNPA1 and DDX5. Genes Dev 32: 1060-1074. doi:10.1101/ gad.316034.118

Lenasi T, Peterlin BM, Dovc P. 2006. Distal regulation of alternative splicing by splicing enhancer in equine $\beta$-casein intron 1. RNA 12: 498-507. doi:10.1261/rna.7261206

Linares AJ, Lin C-H, Damianov A, Adams KL, Novitch BG, Black DL. 2015. The splicing regulator PTBP1 controls the activity of the transcription factor $\mathrm{Pbx} 1$ during neuronal differentiation. Elife 4: e09268. doi:10.7554/eLife.09268

Llorian M, Schwartz S, Clark TA, Hollander D, Tan L-Y, Spellman R, Gordon A, Schweitzer AC, de la Grange P, Ast G, et al. 2010. Position-dependent alternative splicing activity revealed by global profiling of alternative splicing events regulated by PTB. Nat Struct Mol Biol 17: 1114-1123. doi:10.1038/nsmb.1881

Lovci MT, Ghanem D, Marr H, Arnold J, Gee S, Parra M, Liang TY, Stark TJ, Gehman LT, Hoon S, et al. 2013. Rbfox proteins regulate alternative mRNA splicing through evolutionarily conserved RNA bridges. Nat Struct Mol Biol 20: 1434-1442. doi:10.1038/nsmb .2699

Markovtsov V, Nikolic JM, Goldman JA, Turck CW, Chou MY, Black DL. 2000. Cooperative assembly of an hnRNP complex induced by a tissue-specific homolog of polypyrimidine tract binding protein. Mol Cell Biol 20: 7463-7479. doi:10.1128/MCB.20 $.20 .7463-7479.2000$

Mathew R, Hartmuth K, Möhlmann S, Urlaub H, Ficner R, Lührmann R. 2008. Phosphorylation of human PRP28 by SRPK2 is required for integration of the U4/U6-U5 tri-snRNP into the spliceosome. Nat Struct Mol Biol 15: 435-443. doi:10.1038/nsmb .1415

McRae EKS, Booy EP, Moya-Torres A, Ezzati P, Stetefeld J, McKenna SA. 2017. Human DDX21 binds and unwinds RNA guanine quadruplexes. Nucleic Acids Res 45: 6656-6668. doi:10 .1093/nar/gkx380

Min H, Chan RC, Black DL. 1995. The generally expressed hnRNP F is involved in a neural-specific pre-mRNA splicing event. Genes Dev 9: 2659-2671. doi:10.1101/gad.9.21.2659

Miyazaki M, Miyazaki K, Chen K, Jin Y, Turner J, Moore AJ, Saito R, Yoshida K, Ogawa S, Rodewald H-R, et al. 2017. The E-Id protein axis specifies adaptive lymphoid cell identity and suppresses thymic innate lymphoid cell development. Immunity 46: 818-834.e4. doi:10.1016/j.immuni.2017.04.022

Murre C, McCaw PS, Baltimore D. 1989. A new DNA binding and dimerization motif in immunoglobulin enhancer binding, daughterless, MyoD, and myc proteins. Cell 56: 777-783. doi:10.1016/ 0092-8674(89)90682-X

Nakata D, Nakao S, Nakayama K, Araki S, Nakayama Y, Aparicio S, Hara T, Nakanishi A. 2017. The RNA helicase DDX39B and its paralog DDX39A regulate androgen receptor splice variant ARV7 generation. Biochem Biophys Res Commun 483: 271-276. doi:10.1016/j.bbrc.2016.12.153

Pfurr S, Chu Y-H, Bohrer C, Greulich F, Beattie R, Mammadzada K, Hils M, Arnold SJ, Taylor V, Schachtrup K, et al. 2017. The E2A splice variant $E 47$ regulates the differentiation of projection neurons via p57(KIP2) during cortical development. Development 144: 3917-3931. doi:10.1242/dev.145698

Rooke N, Markovtsov V, Cagavi E, Black DL. 2003. Roles for SR proteins and hnRNP $A 1$ in the regulation of c-src exon N1. Mol Cell Biol 23: 1874-1884. doi:10.1128/MCB.23.6.1874-1884 .2003

Sharma S, Falick AM, Black DL. 2005. Polypyrimidine tract binding protein blocks the $5^{\prime}$ splice site-dependent assembly of U2AF and the prespliceosomal E complex. Mol Cell 19: 485-496. doi:10.1016/j.molcel.2005.07.014

Sharma S, Kohlstaedt LA, Damianov A, Rio DC, Black DL. 2008. Polypyrimidine tract binding protein controls the transition from exon definition to an intron defined spliceosome. Nat Struct Mol Biol 15: 183-191. doi:10.1038/nsmb.1375

Sharma S, Maris C, Allain FH-T, Black DL. 2011. U1 snRNA directly interacts with polypyrimidine tract-binding protein during splicing repression. Mol Cell 41: 579-588. doi:10.1016/j.molcel.2011.02 .012

Smith CW, Nadal-Ginard B. 1989. Mutually exclusive splicing of $\alpha$ tropomyosin exons enforced by an unusual lariat branch point location: implications for constitutive splicing. Cell 56: 749-758. doi:10.1016/0092-8674(89)90678-8

Spellman R, Llorian M, Smith CWJ. 2007. Crossregulation and functional redundancy between the splicing regulator PTB and its paralogs nPTB and ROD1. Mol Cell 27: 420-434. doi:10.1016/j molcel.2007.06.016

Sun XH, Baltimore D. 1991. An inhibitory domain of E12 transcription factor prevents DNA binding in E12 homodimers but not in E12 heterodimers. Cell 64: 459-470. doi:10.1016/0092-8674(91) 90653-G

Verma SP, Das P. 2018. G-quadruplex structure at intron 2 of TFE3 and its role in Xp11.2 translocation and splicing. Biochim Biophys Acta 1862: 630-636. doi:10.1016/j.bbagen .2017.11.011

Wagner EJ, Garcia-Blanco MA. 2001. Polypyrimidine tract binding protein antagonizes exon definition. Mol Cell Biol 21: 32813288. doi:10.1128/MCB.21.10.3281-3288.2001 
Wang ET, Sandberg R, Luo S, Khrebtukova I, Zhang L, Mayr C, Kingsmore SF, Schroth GP, Burge CB. 2008. Alternative isoform regulation in human tissue transcriptomes. Nature 456: 470476. doi:10.1038/nature07509

Weldon C, Dacanay JG, Gokhale V, Boddupally PVL, BehmAnsmant I, Burley GA, Branlant C, Hurley LH, Dominguez C, Eperon IC. 2018. Specific G-quadruplex ligands modulate the alternative splicing of Bcl-X. Nucleic Acids Res 46: 886-896. doi:10.1093/nar/gkx1122
Wongpalee SP, Vashisht A, Sharma S, Chui D, Wohlschlegel JA, Black DL. 2016. Large-scale remodeling of a repressed exon ribonucleoprotein to an exon definition complex active for splicing. Elife 5: e19743. doi:10.7554/eLife.19743

Yamazaki T, Liu L, Lazarev D, Al-Zain A, Fomin V, Yeung PL, Chambers SM, Lu C-W, Studer L, Manley JL. 2018. TCF3 alternative splicing controlled by hnRNP H/F regulates E-cadherin expression and hESC pluripotency. Genes Dev 32: 1161-1174. doi:10.1101/gad.316984.118 

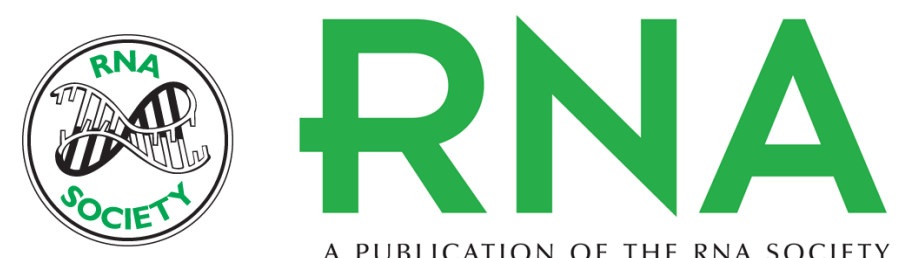

A PUBLICATION OF THE RNA SOCIETY

\section{TCF3 mutually exclusive alternative splicing is controlled by long-range cooperative actions between hnRNPH1 and PTBP1}

Takashi Yamazaki, Lizhi Liu and James L. Manley

RNA 2019 25: 1497-1508 originally published online August 7, 2019

Access the most recent version at doi:10.1261/rna.072298.119

References This article cites 50 articles, 18 of which can be accessed free at: http://rnajournal.cshlp.org/content/25/11/1497.full.html\#ref-list-1

Creative This article is distributed exclusively by the RNA Society for the first 12 months after the Commons full-issue publication date (see http://rnajournal.cshlp.org/site/misc/terms.xhtml). After 12 License months, it is available under a Creative Commons License (Attribution-NonCommercial 4.0 International), as described at http://creativecommons.org/licenses/by-nc/4.0/.

Email Alerting Receive free email alerts when new articles cite this article - sign up in the box at the Service top right corner of the article or click here. 